\title{
Spinal injections under the control of computed tomography with laser navigation
}

\begin{abstract}
The paper presents the author's experience regarding the use of computed tomography with laser navigation in the treatment of lumbar spine and sacroiliac joint pain syndromes. Injections were preformed intra-discally, periarticularly and periradicularly using a mixture of ozone, steroids and local anesthetics, as well as autologous conditioned serum, i.e. Orthokine therapy. Due to the very high efficiency of precise positioning of the needle, the procedure plays a key role in the so-called diagnostic injections in patients at the borderline of surgical treatment.
\end{abstract}

Volume 2 Issue 2 - 2018

\author{
Piotr Godek \\ Sutherland Medical Center, Poland
}

Correspondence: Piotr Godek, Sutherland Medical Center, Poland, Email piotrgodek.smc@gmail.com

Received: March 25, 2018 | Published: April 03, 2018

\section{Introduction}

Disorders of the spine are a civilization problem, a kind of epidemic of our time, not only because of its spread and population range, but also its occurrence at an increasingly young age. It is assumed that $75-85 \%$ of the population complains of pain at the back of the spine, at least once in its lifetime (life-related morbidity), and about 3-5\% of the population complains of radicular pain., ${ }^{1,2}$ The rate of lumbar spine pain is also influenced by the degree of industrialization of the society, it turns out that in populations of highly developed countries this percentage is significantly higher. ${ }^{3}$ Over $90 \%$ of cases of pain in the lumbar spine are included in the group of so-called non-specific mechanical origin. Among them, degenerative-overstrain changes associated with intervertebral disc degeneration (Degenerative Disc Disease, DDD) and the consequences of intersegmental instability and traumatic changes prevail.

The remaining $10 \%$ of cases are diseases related to congenital and developmental defects, metabolic, infectious, neoplastic diseases and inflammatory diseases with autoimmune background. In various age ranges, a change in the percentage share of individual pathologies is noticeable. ${ }^{4}$ It is widely recognized that in the pathogenesis of degenerative changes in the spine (spondylosis), irrespective of the segment - the primary role is played by premature damage to the intervertebral disc. ${ }^{5}$ The consequence of this fact is the intersegmental instability and the intervertebral joints decay being the source of axial pain, and in further consequences the narrowing of the intervertebral foramina and the spinal canal itself, which causes radicular pain and neurological deficits. ${ }^{6}$ Treatment of spodylosis and DDD involves a wide range of procedures ranging from conservative to surgical treatment.

An interesting option of treatment are mini-invasive techniques used in a very dynamically developing area of pain medicine, aimed at alleviating symptoms, especially in chronic phase of spinal pain when none of myriad of rehabilitation methods including physical and manual therapies are insufficient. No matter how sophisticated procedure-spinal injections, mini-destructive procedures (cryoablations, thermoablations, ozone discolysis), implantation of electrodes and spinal cord stimulators or epiduroscopy with root neurolysis each requires precise imaging so that it can be carried out safely and effectively. The most widespread method are however spinal injections guided by one of the form of imaging techniques such as fluoroscopy, USG, CT and MRI. Individual techniques have their important advantages and disadvantages. Fluoroscopy is probably the most widespread method in the world of specialists dealing in pain medicine. It is relatively cheap, easy available and the images are fairly simple to interpret, but it is associated with negative effects of X-rays for both the patient and the operator. In the case of very difficult conditions, for example, for aminal stenosis in the lumbar region or the presence of implants, the location of a needle often requires a series of exposures in several planes. Thus, despite the fact that the dose of radiation in a single CT scan is several times greater than in a single X-ray, the total dose of several exposures may be approaching that achieved in CT. The exposure of the personnel to radiation reflected from the patient's body is also significant. In some cases, the final confirmation of the correct location of the needle is the way of spreading the contrast before the administration of the appropriate drug, which involves additional risk (allergies), and also extends the entire procedure. However, the positioning efficiency of the needle is not impressive at all. In the work of Galholm et al. ${ }^{8}$ The effectiveness of periradicular injections in the lumbar region was estimated at $78 \%{ }^{8}$

Ultrasonography, which is a cheap, repeatable and easily available method, allows even in the presence of moderated degenerative changes and deformations of the spine to precisely perform periarticular injections in the lumbar region (e.g. diagnostic block of the medial branch) at the level of CT control accuracy. ${ }^{9}$ Unfortunately, intraosseous sacroiliac injections and periradicular injections from the transforaminal approach are already burdened with a significant error of inaccuracy, as the target points hide in the acoustic shadow of bone structures. In the presence of implants, ultrasounds are reflected from them and the image becomes illegible. The quality of the image is also affected by the condition of the subcutaneous tissue and fascia density, which causes that in very obese patients the image quality drops sharply and makes the needle difficult to locate even using high quality equipment. The method also does not allow precise location of the needle in the intervertebral space, but it is technically possible to puncture the intervertebral disc under ultrasound control from the lateral access, but both the position of the tip of the needle will not be visible directly or the spread of the drug or gas (i.e. ozone). Despite these problems, ultrasonography turns out to be very useful in everyday practice, due to availability, speed and simplicity of implementation. ${ }^{10}$

Magnetic resonance imaging is a very precise study, but in the case of sever spine proliferative changes, it is often not as precise in the assessment of the size of intervertebral foramina or the intervertebral 
space as the CT examination in the so called bone window. Often in the author's practice, the initial qualification for the procedure of ozone discolysis had to be changed after CT scanning in the bone window, as the intervertebral space turned out to be residual. In addition, injection under MRI control requires the use of special titanium needles, which significantly increases the cost of an expensive and time-consuming study. The presence of implants greatly worsens the quality of the image, and in some cases, even prevents its interpretation. Often, the propagation of the drug around the root must be confirmed by the administration of a gadolinium contrast, which is associated with the risk of allergic reactions. ${ }^{11,12}$ Computed tomography in the so-called bone window seems to be the optimal solution, because it allows very precise determination of bone contours, and thus accurate reproduction of often very tight, crevicular intervertebral spaces, foramina and spinal canal in order to design the access route. The advantage of the method is also the lack of exposure of the personnel to radiation, and the scanning conditions can be selected so that the dose of radiation for the patient will be significantly reduced. The advantage of this imaging is also the fact that in the $3 \mathrm{D}$ reconstruction it is possible to precisely determine the position of the needle and, if necessary, correct its position. This is of great importance in periradicular injections from transformational access, intradiscal injections and of course by epidural injections in the presence of severe spinal stenosis. The method also allows performing an injection in the presence of implants, which, with the appropriate test methodology, only slightly weaken the image quality. In laser-guided devices, a special arm coupled with a gentry's movement guides a laser beam which is actually a predetermined cross-section of the needle approach path, hence the pre-scan can be used to determine the needle's path, angle of needle propagation, distance from the skin to the target, and the point of skin penetration is indicated by a laser marker. The method provides excellent accuracy on the condition of good cooperation with the patient - the patient in the phase between scanning and setting the puncture point cannot move - which is not always easy in patients suffering from pain. In the studies of Moser et al. ${ }^{13}$ The accuracy of the puncture in relation to the planned point reaches $0.5 \mathrm{~mm}$ in relation to $3 \mathrm{~mm}$ in the conventional technique. ${ }^{13}$ In the author's practice, the GE Optima CT660 CT apparatus was used with the Amedo - LNS laser navigation made available in the Innovative Medical Center in Warsaw (Figure 1).

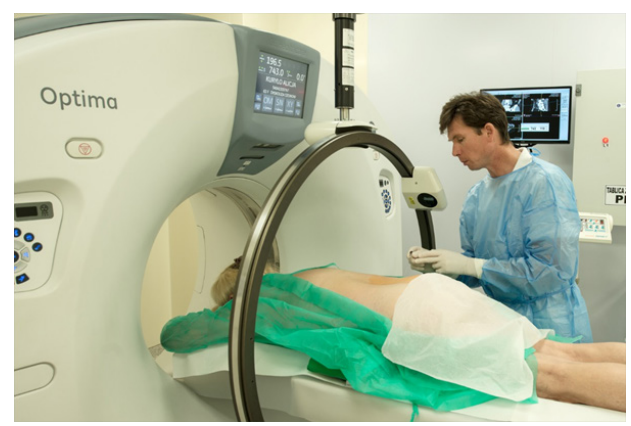

Figure I CT device with laser navigation system.

\section{Materials and results}

\section{Ozone discolysis under CT control}

The treatment consists in administering a mixture of 3-4 ml of ozone gas in a concentration of 30 micrograms per liter to the intervertebral disc. In the author's practice, the ATO-3 ozone generator from Metrum Cryoflex was used. Ozone with anti-inflammatory and antiseptic properties (antibacterial, antifungal and antiviral) also has a strong hygroscopic effect, hence the accelerated breakdown of proteoglycans of the nucleus pulposus and its rapid dehydration with a decrease in its volume and intradiscal pressure, which is one of the important causes of pain. ${ }^{14}$ The treatment is therefore used in the conservative treatment of intervertebral disc injuries, where the goal is to temporarily decompress root-disc conflict and stop the instability of the disc being aware of its irreversible damage. Indications for the discolysis are made after the analysis of MRI images in correlation with the clinical examination. The best indication is the phase of the bulging or early protrusion with the retention of the annulus fibrosus (even in its peripheral zone), with minor degenerative changes of the joints and the lack of spinal stenosis, because significant narrowing of the intervertebral space after discolysis is to be expected. The borderline indication is an extrusion of the intervertebral disc in the MRI image, where the material of nucleus pulposus has not yet been sequestered (also a recurrent postoperative hernia)-many publications have shown that it is still possible to apply the method also in this phase. ${ }^{15-17}$

Contraindications to the procedure are:

a. Extrusion with sequestration

b. Neurological losses

c. Stenosis of the spinal canal

d. Massive degenerative changes of the facet joints

e. Ozone allergy

f. Lack of cooperation with the patient

\section{When to suggest ozone discolysis?}

A. Subacute phase - ineffective conservative therapy lasting 4-6 weeks (rehabilitation, manual therapy, traction table, pharmacotherapy, etc.)

B. A diagnostic injection test (steroid with a local anesthetic) is very helpful in predicting the course of treatment. The interpretation of the test is based on the observation of the degree of pain reduction. A good response, i.e. more than 50\% improvement after 48 hours from injection, means that the swelling of the root dominates as a pain generator and it is possible to continue the attempt of conservative treatment, because usually after reduction of edema in foraminal surrounding the root regains his neuromechanics with the usage of appropriate manual therapy (neuromobilisation). Insufficient answer (lack or very weak improvement), means that the problem is more of mechanical nature and the further compression of the root threatens root ischemia

C. Chronic phase - exacerbation of pain or relapse of symptoms despite the use of conservative treatment

\section{The technique of ozone discolysis under the control of CT with navigation}

The patient placed on the abdomen, the roller under the belly at the place of planned injection should slightly widen the intervertebral space. The first scan (so-called Scout view) ensures the planning of the procedure. After the chosen area scan is performed, the needle 
path is planned in the target of intervertebral space. Subsequently, a $0.7 / 120 \mathrm{~mm}$ puncture needle ( $22 \mathrm{G}$ Braun) is punctured under the surface anesthesia at the exact location of the laser point - ideally in the patient's exhalation phase. After contact with the resistance of the annulus fibrosus, a control scan and $3 \mathrm{D}$ reconstruction are performed to determine the accuracy of the puncture. If the correct location of the needle and its path is confirmed, the steroid (diprophos) and the local anesthetic (bupivacaine) are administered. After a while, the needle is inserted into the central part of the disc and a syringe containing $20 \mathrm{ml}$ of the ozone mixture at a concentration of 30 micrograms per liter is connected to the needle. Gas is slowly injected until sense of resistance of the piston (typically $3-5 \mathrm{ml}$ ), the remaining volume of gas is left on the side wall of the disc and the vertebral body as an additional anti-inflammatory and oxygenating effect of the tissue. After placing the dressing, the patient in the lying position is taken to the observation room, where he spends 2-6 hours depending on the need. The patient is discharged with the recommendation to rest 48 hours and avoid prolonged sitting for the next 2 weeks after the procedure (Figure 2).

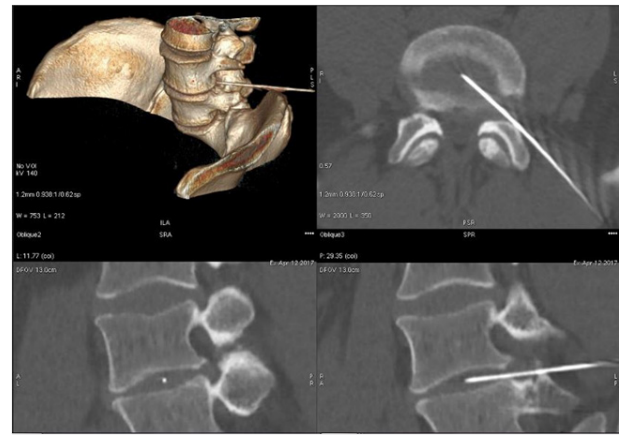

Figure 2 Ozone discolysis level L4 / L5.

\section{Periradicular injection under CT control}

The treatment aims to reduce the edema of the entrapped root, to cut off the pain pertaining to the dorsal ganglia of the spinal nerve and the spinal cord, as well as local oxygenation of the tissues. In view of the above assumptions, the procedure uses a triad of medications - ozone, local anesthetic and steroid. Very often, in the face of severe degenerative changes and foraminal stenosis with borderline indications for conservative treatment, this procedure performs the function of diagnostic injection and is an option for possible further therapy like biologic treatment with Autologic Conditioned Serum (ACS) if it is successful. ${ }^{18}$ The treatment can be repeated after a few months and the cumulative value of repeated injection in long-term observations has been proved. ${ }^{19}$

\section{Technique of periradicular injection under CT control with laser navigation}

The position of the patient and the preparation of the needle path is the same like in the case of ozone discolysis with the difference that now the target of the needle is the intervertebral foramen. After contact of the needle with the root, the patient usually signals this fact very clearly, however, in cases of intensified proliferative degenerative changes, the position of the needle must be confirmed by the control scan and 3D reconstruction. Then after aspiration a mixture of steroid and local anesthetics is administered slowly, observing the patient's condition, and after a while a mixture of ozone gas in a volume of $10-20 \mathrm{ml}$ is delivered depending on the patient's tolerance. The patient should be informed about the temporary loss of motor function and the possibility of a temporary increase in the discomfort due to the volume effect (gas pressure). For the sake of safety after 2-6 hours of observation, the patient always leaves the clinic accompanied by family members. Check-up visit is usually set after 2-3 weeks. In case of a clear improvement, it is possible to continue the treatment with ACS to maintain the anti-inflammatory effect (Figure 3).

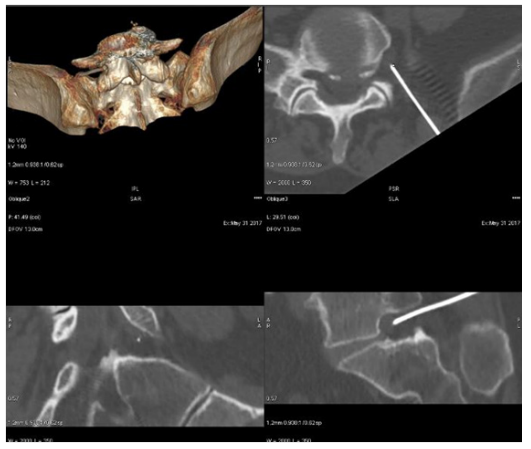

Figure 3 Periradicular injections under CT control with laser navigation of the L5SI level.

\section{ACS (Orthokine) treatment under CT control}

The principle of ACS activity is competitive blocking of the Interleukin-1 receptors through specific proteins are their ligand (IL-1Ra protein). After incubating the patient's own blood in special EOT II tubes containing spheres of chromium-coated glass, there is a dramatic increase in the concentration of IL-1Ra (even 140x more than in normal conditions) and a significant increase in active growth factors. Thus, the serum has a very strong and long-lasting antiinflammatory and regenerative effect. It is possible to administer ACS, periarticularly, intraarticularly, periradicularly and even epidural in up to 6 doses of $3-4 \mathrm{ml}^{20,21}$

\section{Indications}

a. herniation and dehydration of the intervertebral disc in the MRI image with the continuity of the fibrous annulus - possible intradiscal administration - the best indication, especially in young people, the aim of the treatment is to inhibit the progression of the degenerative intervertebral disc

b. an intervertebral disc herniation in an MRI image with a root compression, where the nucleus pulposus fragmentation has not yet been sequestered (also a recurrent postoperative hernia)

c. multilevel degenerative changes of the intervertebral joints, degenerative scoliosis, postoperative conditions (inflammation, scars) - the periarticular and epidural route

\section{Contraindications}

a. Fever

b. Active infection

c. Period of 1 month after vaccination

The injection technique is identical to the one described above, however, it should be remembered that intradiscally is delivered only part of the dose obtained after centrifugation with $10 \mathrm{ml}$ of whole blood, i.e. approx. $2-3 \mathrm{ml}$, the rest of the material is distributed periarticularly. In the period 11/2016-12/2017 the following 
procedures were performed in a total group of 98 patients (women 55 , men 43)

Ozone discolysis-23 patients, Periradicular ozone injection-60 patients, ACS therapy (Orthokine)-15 patients (including intradiscal-1, SI-joint -1, periradicular -13), Patients' age was 18 to 92 years, mean age 63.70. Totally 91 patients were evaluated during check- up and the results were assessed on the modified Mcnab scale, asking for the determination of the result in 4 given categories as below (Figure 4).

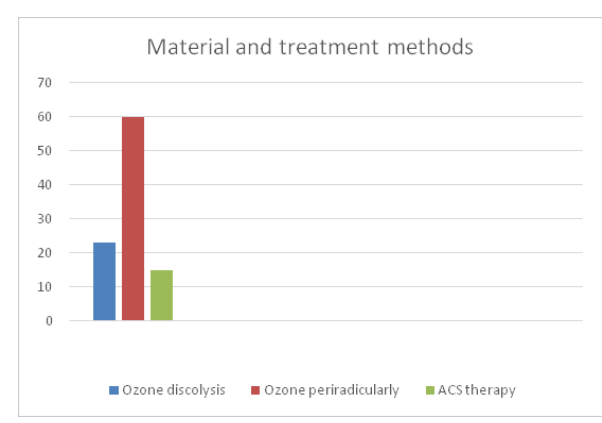

Figure 4 Material and treatment methods.

\section{Modified Mcnab scale}

How would you describe the overall result after ozone discolysis, ozone injection or ACS therapy performed under CT control?

i. Excellent - no pain, no mobility restrictions, no return to current work and level of activity

ii. Good - occasionally pain occurs but does not radiate to the leg and the pain is much weaker and it is possible to return to the current work and activity

iii. Average - slight improvement, slight pain reduction, but still impossible to return to work and previous activity

iv. Bad - no improvement, still significant pain, impossible return to work and activity, or there was a need for surgical treatment ${ }^{22}$ (Figure 5).

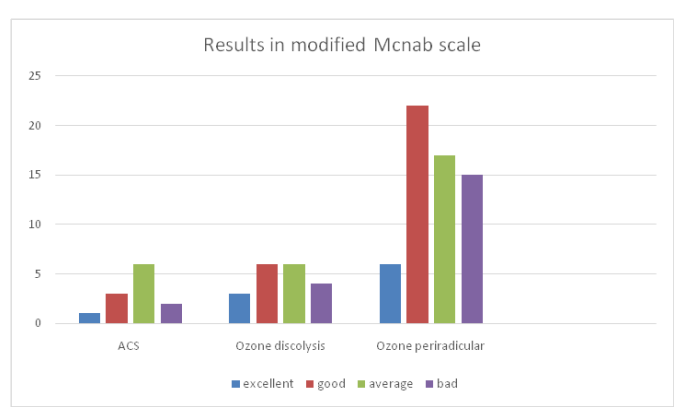

Figure 5 Results in modified Mcnab scale.

\section{Discussion}

The group of patients undergoing control in the ACS therapy is too small to draw far-reaching conclusions. It should be noted, however, that patients with chronic pain who failed other treatment methods were qualified, and in 2 cases patients after extensive lumbar spine stabilization. In this group, 1 injection in a young patient with intervertebral disc injury in the bulging/hernia stage was performed, however, it did not obtain a satisfactory result in the 2-month follow-up, 1 injection into the sacroiliac joint and 10 periradicular injections in patients with a sever degenerative changes, obtaining 3 good results, 6 average and only $1 \mathrm{bad}$. In the case of the patient's qualification for the intradiscal administration, several pilot studies were based on interesting results regarding the chances of stopping the degeneration of the intervertebral disc at an early stage of the process by administering biologically active substances such as platelet rich plasma (PRP) or autologous conditioned plasma (ACS). ${ }^{23-25}$ In the ozone discolysis group the L4/L5 disc protrusion predominated in this group, although only half of the patients obtained a satisfactory result, it should be emphasized that only 3 patients had to undergo surgical treatment, the rest despite the average result could undertake further rehabilitation that would restore their functional status. In the publications of Italian authors having the largest material of patients undergoing ozone discolysis, about $70 \%$ of patients report a positive result, and the addition of steroid periradicularly during the procedure shows a cumulative effect still improving results compared to the group of ozone itself. ${ }^{26}$

Ozone periradicular injection group - the most numerous in our material and the most inhomogeneous, because in addition to the rootdisc conflict in the presence of intervertebral disc hernia, patients also demonstrated critical spinal canal stenosis, spondylolytic instability or degenerative scoliosis with massive degenerative-proliferative changes of the facet joints. In this group there were cases qualified for surgery, and the procedure was often treated as a symptomatic treatment. Nevertheless, in half of the cases a satisfactory result was obtained allowing further rehabilitation or injection therapy (collagen, serum, etc.) and only $25 \%$ of patients assessed this procedure unambiguously negatively and 3 patients in this group had to undergo surgical treatment. Taking into consideration the advanced age of treated patients and the severity of degenerative changes in this group, which is very often a borderline condition for conservative treatment - the above-mentioned, not too spectacular results should be interpreted with some reserve. The procedure has also an additional diagnostic value. After achieving improvement even partially, one can hope that the continuation of conservative treatment will have a chance of success. Although the literature about periradicular steroid injection is very rich and confirms their effectiveness, Benetti et al. ${ }^{29}$ showed a comparable effect of periganglionic injections made using only ozone, yielding over $78 \%$ of satisfactory results. ${ }^{27-29}$

\section{Conclusion}

I. Computed tomography in the bone window and $3 \mathrm{D}$ reconstruction has a significant advantage over other forms of spinal injections guidance

II. Laser navigation combined with computed tomography image, especially in $3 \mathrm{D}$ reconstructions, allows very precise placement of the needle even in very difficult conditions related to severe degenerative changes and in the presence of postoperative implants

III. Diagnostic injections with the precision that CT provides with navigation using the triad-ozone, steroid, local anesthetic are a very effective prognostic factor and guide for further treatment

IV. Therapy with ACS seems to be an interesting option in further anti-inflammatory therapy in patients with a clear improvement after a diagnostic injection. 


\section{Acknowledgements}

None.

\section{Conflict of interest}

Author declares that there is no conflict of interest.

\section{References}

1. Nachemson AL, Jonsson E. Annual spine disease varies from 15 to $20 \%$ in the United States and from 25 to $45 \%$ in Europe. Neck and back pain, Philadelphia: Wiliams \& Wilkins; 2000

2. Andersson GBJ. Epidemiology of the low back pain. Acta Ortho Scand. 1998;69(S281):28-31.

3. Borenstein D. Epidemiology, etiology, diagnostic evaluation and treatment of low back pain. Curr Opin Rheumatol. 1998;10(2):104-9.

4. Nguyen TH, Randolph DC. Nonspecific low back pain and return to work. Am Fam Physician. 2007;76(10):1497-502.

5. Boni M, Denaro V. Anatomic-clinical correlations in cervical spondylosis In: Kehr P, Weidner A, editors. Cervical spine I. New York: SpringerVerlag; 1987. p. 3-20

6. Hoppenfeld JD. Fundamentals of Pain Medicine: How to Diagnose and Treat your Patients. Wolters Kluwer: 2014.

7. Hooten WM, Cohen SP. Evaluation and Treatment of Low Back Pain A Clinically Focused Review for Primary Care Specialists. Mayo Clin Proc. 2015;90(12):1699-718.

8. Galhom AE, Shatouri MA. Efficacy of therapeutic fluoroscopy-guided lumbar spine interventional procedures. Clin Imaging. 37(4):649-56.

9. Galiano $\mathrm{K}$, Obwegeser AA, Walch $\mathrm{C}$, et al. Ultrasound-guided versus computed tomography-controlled facet joint injections in the lumbar spine: a prospective randomized clinical trial. Reg Anesth Pain Med. 2007;32(4):317-22.

10. Loizides A, Peer S, Plaikner M, et al. Ultrasound-guided injections in the lumbar spine. Med Ultrason. 2011;13(1):54-8.

11. Fritz J, Sequeiros RB, Carrino JA. Magnetic resonance imaging-guided spine injections. Top Magn Reson Imaging. 2011;22(4):143-51.

12. Fritz J, Thomas C, Clasen S. Freehand real-time MRI-guided lumba spinal injection procedures at $1.5 \mathrm{~T}$ : feasibility, accuracy, and safety. AJR Am J Roentgenol. 2009;192(4):W161-7.

13. Moser C, Becker J, Deli M. A novel Laser Navigation System reduces radiation exposure and improves accuracy and workflow of CT-guided spinal interventions: a prospective, randomized, controlled, clinica trial in comparison to conventional freehand puncture. Eur J Radiol. 2013;82(4):627-32.

14. Zhang Y, Ma Y, Jiang J, et al. Treatment of the lumbar disc herniation with intradiscal and intraforaminal injection of oxygen-ozone. $J$ Back Musculoskelet Rehabil. 2013;26(3):317-22.
15. Bocci V, Borrelli E, Zanardi I, et al. The usefulness of ozone treatment in spinal pain. Drug Des Devel Ther. 2015;9:2677-85.

16. Lu W, Li YH, He XF. Treatment of large lumbar disc herniation with percutaneous ozone injection via the posterior-lateral route and inner margin of the facet joint. World J Radiol. 2010;2(3):109-12.

17. Magalhaes FN, Dotta L, Sasse A, et al. Ozone therapy as a treatment for low back pain secondary to herniated disc: a systematic review and meta-analysis of randomized controlled trials. Pain Physician. 2012;15(2):E115-29.

18. Quraishi NA. Transforaminal injection of corticosteroids for lumbar radiculopathy: systematic review and meta-analysis. Eur Spine J. 2012;21(2):214-9.

19. Murthy NS, Geske JR, Shelerud RA, et al. The effectiveness of repeat lumbar transforaminal epidural steroid injections. Pain Med. 2014;15(10):1686-94.

20. Godek P. Use of Autologous Serum in Treatment of Lumbar Radiculopathy Pain. Pilot Study. Ortop Traumatol Rehabil. 2016;18(1):11-20.

21. Becker C, Heidersdorf S, Drewlo S, et al. Efficacy of epidural perineural injections with autologous conditioned serum for lumbar radicular compression: an investigator-initiated, prospective, double-blind, reference-controlled study. Spine. 2007;32(17):1803-8.

22. Macnab I. Negative disc exploration: an analysis of the cause of nerve root involvement in sixty-eight patients. J Bone Joint Surg Am. 1971;53:891-903.

23. Wang SZ, Rui YF, Tan Q, et al. Enhancing intervertebral disc repair and regeneration through biology: platelet-rich plasma as an alternative strategy. Arthritis Res Ther. 2013;15(5):220.

24. Akeda K, Ohishi K, Masuda K, et al. Intradiscal Injection of Autologous Platelet-Rich Plasma Releasate to Treat Discogenic Low Back Pain: A Preliminary Clinical Trial. Asian Spine J. 2017;11(3):380-389.

25. Moser C, Groenemeyer DD, Becker $\mathrm{J}$, et al. Wehling Intradiscal Injections of Orthokine -derived Autologous Conditioned Serum (ACS) for Lumbar Disc Degeneration. Osteoarthritis and Cartilage. 19:S215.

26. Andreula CF, Simonetti L, Santis F, et al. Minimally invasive oxygenozone therapy for lumbar disk herniation. AJNR Am $J$ Neuroradiol. 2003;24(5):996-1000.

27. Bui J, Bogduk N. A systematic review of the effectiveness of CTguided, lumbar transforaminal injection of steroids. Pain Med. 2013;14(12):1860-5.

28. Vicar J, King W, Landers MH, et al. The effectiveness of lumbar transforaminal injection of steroids: a comprehensive review with systematic analysis of the published data. Pain Med. 2013;14(1):14-28.

29. Bonetti M, Fontana A, Cotticelli B, et al. Intraforaminal $\mathrm{O}(2)-\mathrm{O}(3)$ versus periradicular steroidal infiltrations in lower back pain: randomized controlled study. AJNR Am J Neuroradiol. 2005;26(5):996-1000. 